Original Article

\title{
Effect of different hand positions on trunk and shoulder kinematics and reaction forces in sitting pivot transfer
}

\author{
Sung Shin KIm ${ }^{1)}$, Jin Gan HeR ${ }^{1)^{*}}$, TAe Sung $\mathrm{Ko}^{2)}$ \\ 1) Department of Rehabilitation Therapy, Graduate School of Hallym University: 1 Hallimdaehak-gil, \\ Chuncheon-si, Gangwon-do, Republic of Korea \\ 2) Department of Physical Therapy, Daewon University College, Republic of Korea
}

\begin{abstract}
Purpose] The purpose of this study was to compare the changes in trunk and shoulder angles, and reaction forces under the two hands elicited by different hand base of support positions during sitting pivot transfer. [Subjects and Methods] Eighteen unimpaired subjects performed independent sitting pivot transfer. Subjects performed sitting pivot transfer between an initial seat to a target seat by only using their hands positioned at the same height as and lower than the seat position. Trunk and shoulder kinematics, and reaction forces on the trailing and leading hands were calculated. Mean peak joint angles and forces were compared between the hand positions using the pared t-test for the lift phase of the transfer. [Results] There were significant increases in the trunk angles of forward and lateral flexion, even though rotation decreased while transferring in the lower hand position. Increased shoulder flexion, anterior/posterior forces and reduced lateral forces were also shown. [Conclusion] Placing the hands of the supporting arms lower than the seat position during sitting pivot transfer was identified as having biomechanical advantages. Therefore, the lower hand position can be recommended as an effective and safe method for sitting pivot transfer by patients with spinal cord injury and can be utilized as a reference data for considering the appropriate height of aids for a wheelchair.

Key words: Hand position, Sitting pivot transfer, Shoulder pain
\end{abstract}

(This article was submitted Mar. 9, 2015, and was accepted Apr. 16, 2015)

\section{INTRODUCTION}

Patients with spinal cord injuries (SCI) have motor and sensory disorders, resulting in disabilities in daily activities ${ }^{1)}$. Although they should receive intensive care and rehabilitation treatment after injury, most patients experience disorders (e.g., impingement syndrome, osteoarthritis, bursitis, paralysis, parasthesia) in their upper extremities, trunk, or lower extremities due to physiological and physical changes. They experience functional disorders in ambulation, particularly with respect to activities of daily living $(\mathrm{ADL})^{2,3)}$. Due to these ambulatory disorders, spinal cord injury patients often use wheelchairs and learn to perform basic ADL (e.g. propulsion, lifting, transfer) in relation to the use of a wheelchair.

These patients often suffer upper extremity pain due to performance of ADL almost exclusively by the upper extremities (overall upper extremity pain prevalence rates: $58.5 \%$; shoulder: $71 \%$, elbow: $35 \%$, wrist: $53 \%$, hand:

*Corresponding author. Jin Gang Her (E-mail: Jghur7@ empas.com)

C 2015 The Society of Physical Therapy Science. Published by IPEC Inc. This is an open-access article distributed under the terms of the Creative Commons Attribution Non-Commercial No Derivatives (by-ncnd) License $<$ http://creativecommons.org/licenses/by-nc-nd/3.0/>.
$43 \%)^{4)}$, and such pain impedes the performance of $\mathrm{ADL}^{5}$. Among the basic ADL, transfer is a task that is performed by each patient $15-20$ times per day, on average. This is the most burdensome task for the musculoskeletal system, the nervous system, and the cardiovascular system among wheelchair related ADL. In particular, transfer imposes the largest load on the shoulder joint ${ }^{6,7)}$.

Sitting pivot transfer (SPT) is the most frequently used transfer method using the upper extremities. Using this method, the hip is moved from the initial seat (Iseat) to the target seat (Tseat) using the trailing hand (TH) and the leading hand (LH) while both lower extremities maintain contact with the floor as an axis ${ }^{6}$. There are largely two main SPT strategies. One is a translational strategy in which the head and the hip move horizontally in the same direction, and the other is a rotational strategy in which the head and the hip move while rotating in different directions using both feet as a pivot ${ }^{2)}$. During SPT movements, the shoulder is in a position of internal rotation and abduction, which will invariably cause shoulder impingement unless other aids are used ${ }^{6}$. Although there are many shoulder pain treatment methods, the shoulder cannot take any rest because of transfer movements, which are essential for patients' ADL. Consequently, shoulder pain leads to various complications ${ }^{5)}$.

Recently, some studies have been conducted of methods for minimizing shoulder injuries through dynamic analyses of transfer movements. According to a recent study, SPT is 
the most efficient and imposes the lowest loads on individual joints when the wheelchair moves horizontally without any variation in floor height compared to when there are differences in height between the position of the wheelchair and the floor on which the wheelchair moves ${ }^{8)}$.

A previous study using kinematic analysis of SPT movements reported that, in an experiment conducted to measure loads on the shoulder joint in three different positions, anterior trunk positions mobilized large muscles around the shoulder and the mobilization of muscles reduced the risk of rotator cuff impingement ${ }^{2}$. The maximum flexor momentum at the shoulder joint during SPT movements is known to be approximately $1.45 \mathrm{Nm} / \mathrm{kg}$ at the $\mathrm{TH}$ and approximately $1.36 \mathrm{Nm} / \mathrm{kg}$ at the $\mathrm{LH}$ of patients and approximately 1.56 $\mathrm{Nm} / \mathrm{kg}$ in healthy adults ${ }^{9}$. Joint reaction force is known to be always higher at $\mathrm{TH}$ than at $\mathrm{LH}$, and joint loads are known to be higher during transfer to higher seats ${ }^{10}$.

In a recent study of human movement, it was reported that the destabilizing force $(\mathrm{dF})$, which tends to make movements, was determined by the height of the center of mass (COM), center of pressure (COP), base of support (BOS), and the reaction force and shear force acting on the floor ${ }^{11)}$. In a study of the $\mathrm{dF}$ and stabilizing force $(\mathrm{sF})$ occurring in SPT movements, it was reported that low $\mathrm{dF}$ and high $\mathrm{sF}$ were shown, and that the low $\mathrm{dF}$ and high $\mathrm{sF}$ were related to the securing of stability against the risk of falls ${ }^{12,13)}$.

Nevertheless, no study to date has been conducted on changes in loads on the lower extremities during actual SPT in relation to decreases in BOS and increases in the height of the COM. Additionally, little is known about the effects of the these changes. Studies of transfer methods that will reduce the burden on the upper extremity will contribute to the relief of shoulder pain eventually leading to the prevention of paraplegic patients' shoulder pain. Therefore, the purpose of the present study was to examine the effects of different heights of the hands of the supporting arms on the loads on the shoulder joint during transfer movements in order to seek effective and safe transfer methods.

\section{SUBJECTS AND METHODS}

The subjects of the present study were recruited from among students, who voluntarily wanted to participate in the study, attending in Hallym College located in Chuncheonsi. Based on the principles of the Declaration of Helsinki, research ethics, the purpose and contents of the study were sufficiently explained to the subjects before they participated in the experiment, and they were required to submit their written informed consent. The subjects had no history of musculoskeletal system disease of their upper extremity. Among the recruited subjects, only 18 adult males participated in the experiment: excluding those who could not lift their trunk because their upper extremity muscle strength was not sufficient to perform SPT movements and those who could not wear the tight suit for motion analysis were excluded.

The Iseat and Tseat of the device specially designed for SPT were made from $30 \times 50 \mathrm{~cm}^{2}$ sized $3.2 \mathrm{~mm}$ thick steel plates, and the plates for TH and LH were made using $60 \times$ $50 \mathrm{~cm}^{2}$ sized steel plates of the same thickness as the seats.
For height adjustment, hand jerks that could endure at least $2,000 \mathrm{kgf}$ were fixed by welding between the steel plates. Thereafter, $1 \mathrm{~cm}$ diameter holes were drilled at the four corners of the welded steel plated using a laser. Long steel bolts were inserted through the holes to fix the upper and lower steel plates of the Iseat and Tseat. The TH and LH plates were fixed with bolts in order to minimize vibration or shaking caused by loads during transfer movements. Then, $20 \mathrm{~cm}$ high aluminum profiles were fastened to the two fixed steel plates using bolts so that the final height could be adjusted to between $35 \mathrm{~cm}$ and $65 \mathrm{~cm}$. All joint areas except for the hand jerks were firmly fastened using bolts.

Each subject performed SPT movements on a special device designed for the experiment. Prior to the experiment, audio-visual education on the rotational strategy among SPT method was conducted along with a verbal explanation. The experiment was conducted following changing the height of the hands. The subjects moved from the Iseat at a height of $50 \mathrm{~cm}$ to the Tseat at the same height at an angle of $90^{\circ}$. The subjects adopted a posture with the TH located as close as possible to the hip on the Iseat and the LH located as close as possible to the Tseat. To keep the same hand positions for all subjects, the shape of a hand was printed and attached to the positions of the TH and the LH on the force plates (Fig. 1).

After the verbal order "Start", the subjects moved from the Iseat to the Tseat by lifting their hips solely using the strength of their arms. At this time, the subjects were instructed to limit the activity of their lower extremities maximally. Then, the subjects performed the same movement after reducing the height of the $\mathrm{TH}$ and the $\mathrm{LH}$ plates by $10 \mathrm{~cm}$. The height of 50 or $40 \mathrm{~cm}$ was randomly selected to

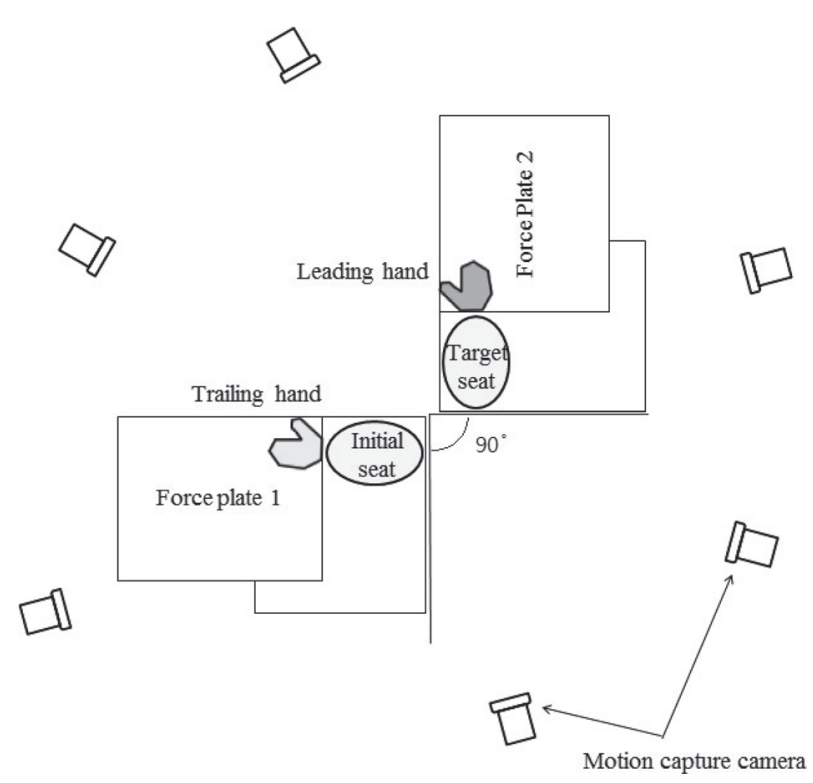

Fig. 1. Two tables that can be adjusted in height and two force plates

Six motion analysis cameras were placed around the device. The trailing hand and the leading hand were placed on the hand shaped marks attached to the force plates and the initial seat and the target seat were placed immediately next to the two hand positions. The angle between the two tables was set to $90^{\circ}$ 
eliminate order effects. Each movement was measured five times repeatedly at the two heights. The subjects took a sufficient rest of one minute between each movement. During the SPT movements, if a subject complained of pain in any part of the upper extremity, the experiment was immediately stopped.

In the present study, SPT movements were divided into two events: the start and ending events. The time labeled at which loading occurred on the force plate below the $\mathrm{LH}$ after the verbal order "Start" was labeled event1, and the time at which the loading became 0 at the TH plate, when the transfer to the Tseat had been completed, was labeled event2.

The angles of the movements of the upper extremity and the trunk were measured using a motion analyzer (Oqus100, Qualisys, Sweden). Before the experiment, the study subjects wore upper and lower body stockings that clung to their body shapes, and $15 \mathrm{~mm}$ diameter reflective markers, that could be recognized by infrared cameras, were attached to the $\mathrm{C} 7$ and T10 vertebrae, jugular notch, xiphoid process of the sternum, head (left/right forehead and rear head), acromion, upper arms, humeral lateral epicondyles, wrist lateral/ medial epicondyles of the wrists, 2nd metacarpal heads, and ASIS and PSIS. The light signals reflected individual markers were recorded using six cameras and QTM (Qualisys, Sweden) at a rate of 100 frames per second. The body joints and segments in the obtained data were labeled, and the kinematics of individual joints and segments were quantitatively analyzed using Visual 3D software (C-Motion, USA). To reduce measurement errors, the markers for motion analysis were all attached by one person.

Force plates (9260AA6, Kistler, Switzerland) were used to measure the external force generated by the upper extremities while SPT movements were being performed. The voltages measured at different loads were amplified through an amplifier (5233A2, Kistler, Switzerland) and transmitted to the computer through an A/D board (USB-2533, Measurement Computing Corporation, USA). Then, the values of the force applied to the force plates by external loads were calculated using QTM motion analysis software program. Kinetic data were sampled at $1,000 \mathrm{~Hz}$ to align with the kinematic data. The kinematic data measured between event1 and event 2 of each subjects were evenly divided into 101 frames (0 to 100 frames) and normalized to each subject's height and weight values. Each subject's SPT movements were measured five times and the average was used in the analysis.

Statistical analysis was conducted using PASW ver. 18.0 (SPSS, IBM, USA). The paired t-test was used to compare kinetic and kinematic data between the two heights of the
TH and LH. The significance level, $\alpha$, was chosen as 0.05 .

\section{RESULTS}

The group means (standard deviation) of age, height, weight of the participants were 21.11 (1.71) years, 1.78 (0.04) meters, 74.28 (9.40) kilograms, respectively.

The trunk movements measured at the different hand heights were as follows. The maximum flexion of the trunk was significantly larger when the hand position was low $75.14(7.95)^{\circ}$ than when the hand position was normal 53.12 $(13.50)^{\circ}(\mathrm{p}=0.000)$. The maximum ranges of lateral flexion when the hand positions were low and normal were 48.04 $(9.37)^{\circ}$ and $42.82(9.74)^{\circ}$, respectively, and the difference was significant $(\mathrm{p}=0.022)$. The maximum ranges of rotation when the hand positions were low and normal were 0.26 $(9.72)^{\circ}$ and $17.89(11.24)^{\circ}$, respectively, and the difference was significant $(\mathrm{p}=0.000$; Table 1$)$.

The maximum flexion angles of the shoulder joint when the hand positions were low and normal were $51.55(10.25)^{\circ}$ and $40.89(11.35)^{\circ}$, respectively, at the $\mathrm{TH}(\mathrm{p}=0.000)$ and $32.03(8.87)^{\circ}$ and $18.68(7.29)^{\circ}$, respectively, at the LH $(\mathrm{p}=0.000)$. The differences in the angles between the two hand heights were significant at both the $\mathrm{TH}$ and at the $\mathrm{LH}$. The maximum abduction angles were not significantly different between the two hand heights at the $\mathrm{TH}$, but showed significant differences at the $\mathrm{LH}$, with values of 15.60 $(10.53)^{\circ}$ and $13.51(8.04)^{\circ}$ when the hand positions were low and normal, respectively $(\mathrm{p}=0.027)$. The maximum rotation angles were not different between the two hand heights at the $\mathrm{TH}$, but they showed significant differences at the $\mathrm{LH}$, with values of $74.79(14.22)^{\circ}$ and $64.90(11.20)^{\circ}$ when the hand positions were low and normal, respectively $(\mathrm{p}=0.000$; Table 2).

The values of anterior/posterior force measured at the force plates below the $\mathrm{TH}$ and $\mathrm{LH}$ when the hand positions were low and normal were 8.98 (1.96)\% (BW) and 7.89 (1.96)\% (BW), respectively, at the $\mathrm{TH}$, and the difference

Table 1. Means $( \pm$ SD) of the peak trunk angles of each hand height

\begin{tabular}{llcc}
\hline \multirow{2}{*}{ Component } & \multicolumn{2}{c}{ Trunk } \\
\cline { 3 - 4 } & & HL & HN \\
\hline \multirow{2}{*}{ Peak } & Flexion & $75.1(8.0)^{*}$ & $53.1(13.5)$ \\
angle & Lateral flexion (left) & $48.0(9.4)$ & $42.8(9.7)$ \\
(degree) & Rotation (left) & $0.3(9.7)^{*}$ & $17.9(11.2)$ \\
\hline
\end{tabular}

HL: low hand height $(40 \mathrm{~cm}), \mathrm{HN}$ : normal hand height $(50 \mathrm{~cm})$, $* \mathrm{p}<0.05$

Table 2. Means $( \pm \mathrm{SD})$ of the peak shoulder angles of each hand height

\begin{tabular}{llllll}
\hline \multirow{2}{*}{ Component } & \multicolumn{2}{c}{ Trailing hand } & \multicolumn{2}{c}{ Leading hand } \\
\cline { 2 - 6 } & \multicolumn{2}{c}{ HL } & HN & HL & HN \\
\hline \multirow{2}{*}{ Peak } & Flexion & $51.6(10.3)^{*}$ & $53.1(13.5)$ & $32.0(8.9)^{*}$ & $18.7(7.3)$ \\
angle & Abduction & $16.6(8.6)^{*}$ & $42.8(9.7)$ & $15.6(10.5)^{*}$ & $13.51(8.0)$ \\
(degree) & Rotation (internal) & $37.5(19.3)^{*}$ & $17.9(11.2)$ & $74.8(14.2)^{*}$ & $64.9(11.2)$ \\
\hline \multicolumn{2}{l}{ HL: low hand height (40 cm), HN: normal hand height $(50 \mathrm{~cm}),{ }^{*} \mathrm{p}<0.05$}
\end{tabular}


was significant $(\mathrm{p}<0.05)$, but there was no significant difference at the LH. The values of lateral/medial force showed significant differences between the two hand heights at the TH with values of $7.96(2.33) \%(\mathrm{BW})$ and $8.92(2.92) \%$ (BW), respectively $(\mathrm{p}<0.05)$, but did not show any significant difference at the LH. The values of vertical force did not show statistically significant difference between the two hand heights at the TH or the LH (Table 3).

\section{DISCUSSION}

The purpose of the present study was to analyze the movements of the shoulder joint and the trunk at different heights of the TH and the LH in SPT movements to provide reference data for effective and safe transfer methods for spinal cord injury, paraplegia, and lower extremity amputation patients. Gagnon et al. reported that during transfer to seats of different heights, trunk flexion of $68-71^{\circ}$ appeared at the initial hip lifting, that trunk flexion facilitated SPT movements during which the hip was lifted and moved, and that trunk flexion affected balance and stability in spinal cord injury patients with weakened erector spinae muscle or neurological disorders with upper extremity muscle strength $^{8)}$. Tanimoto et al. advised that the degree of trunk flexion increased the time of hip lifting for the entire SPT movement ${ }^{14)}$. Finley noted that patients who complained of shoulder impingement showed smaller trunk flexion angles, and that smaller angles were associated with the formation of small trunk flexion moments at the beginning of the initial phase of hip lifting after injury ${ }^{6}$. In the present study, when the hand height was in the lower position $(40 \mathrm{~cm})$, trunk flexion essentially increased to use the lower hand position as the weight-bearing surface. Positive effects could be expected from this in that trunk flexion generally acts as propulsion during hip lifting at the beginning of transfer movements.

Another movement that indispensably appears along with trunk flexion in SPT movements is rotation. Trunk rotation occurs in the opposite direction to the seat that would move around the TH. According to a study conducted by Forslund et al. ${ }^{15)}$, trunk rotation of $41^{\circ}$ can occur at the maximum in females, and $32^{\circ}$ can occur at the maximum in males. The difference was assumed to be attributable to differences in shoulder widths and pelvis sizes between the genders. In the present study, rotation was shown to be smaller by $17.73^{\circ}$ on average when the hand height was lower. This is assumed to be associated with the increase in trunk flexion appearing when the hand height is low. In addition, increases in trunk rotation are thought to affect shoulder joint angles and the strength of the surrounding muscles directly.

In previous SPT movement analysis studies, maximum shoulder joint flexion was $36^{\circ}$, abduction was $50^{\circ}$, and internal rotation was $\left.15-30^{\circ 6}, 16,17\right)$. It is known that during transfer movements, complicated upper extremity movement combinations reduce the subacromial space and eventually affect the rotator-cuff muscle and soft tissues (e.g. bursae) around the shoulder joint resulting in shoulder impingement. The angles of flexion and abduction at low hand positions in the present study were different from those reported by previous studies. Flexion should increase when the bearing surface is low, and larger flexion and abduction angles should occurs to compensate for the bent trunk (Fig. 2).

Since trunk rotation movements occur around the axis of the TH at the beginning, of SPT movements, internal rotation of the TH shoulder is inevitable. In a study conducted by Finely et al., patients with shoulder impingement were identified as utilizing more internal rotation than other patients during SPT movements ${ }^{6}$. Also, a study conducted by Riek et al. reported that internal rotation of $4-9^{\circ}$ occurred at the TH of spinal cord injury patients. In the present study, internal rotation at the $\mathrm{TH}$ occurred more frequently when the hand was low, and this seems to increase the risk of shoulder impingement ${ }^{17)}$. However, Gagnon et al. ${ }^{8)}$ advised that the maximum angle of internal rotation might vary with hand position when SPT movements began. Therefore, this problem might be resolved by studies seeking the hand position that can minimize internal rotation of the shoulder.

Forslund et al. ${ }^{15)}$ reported that when SCI patients transferred to a seat that was $7 \mathrm{~cm}$ higher, the maximum vertical reaction force at the $\mathrm{TH}$ was always higher than that at the $\mathrm{LH}$, and that this seemed to be a natural phenomenon because

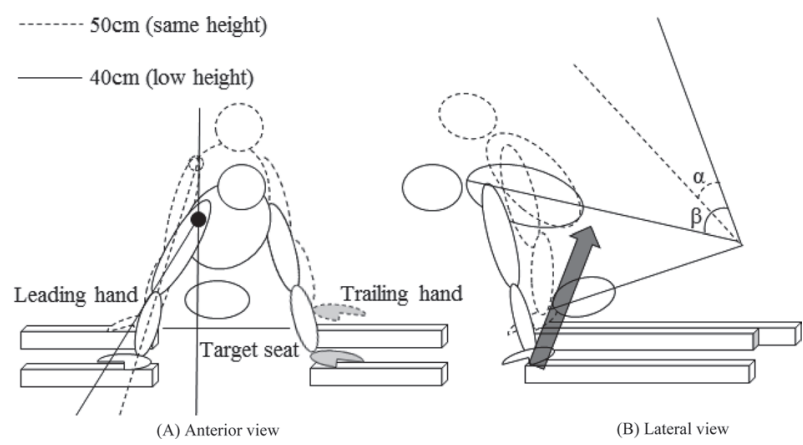

Fig. 2. Changes in shoulder joint and trunk angles according to bearing surface heights

$\alpha$ : trunk flexion angle at $\mathrm{HN}, \beta$ : trunk flexion angle at $\mathrm{HL}$

Table 3. Means ( \pm SD) of the peak forces of each hand height

\begin{tabular}{llrrrr}
\hline \multirow{2}{*}{ Component } & \multicolumn{2}{c}{ Trailing hand } & \multicolumn{2}{c}{ Leading hand } \\
\cline { 2 - 6 } & & \multicolumn{1}{c}{ HL } & \multicolumn{1}{c}{ HN } & \multicolumn{1}{c}{ HL } & \multicolumn{1}{c}{ HN } \\
\hline \multirow{2}{*}{ Peak } & Posterior & $9.0(2.0)^{*}$ & $7.9(1.7)$ & $0.1(0.5)$ & $0.1(0.3)$ \\
Force & Lateral & $8.0(2.3)^{*}$ & $8.9(2.9)$ & $1.4(1.8)$ & $1.3(0.8)$ \\
$(\% \mathrm{BW})$ & Vertical & $44.7(6.0)$ & $45.4(4.9)$ & $43.2(7.2)$ & $45.2(6.5)$ \\
\hline
\end{tabular}

BW: body weight, ${ }^{*} \mathrm{p}<0.05$ 
the TH is closer to the body to initiate movements at the beginning of the movement. They reported that the average vertical reaction force at the TH was $32 \%(\mathrm{BW})$, and that it was $24.5 \%$ (BW) at the LH. In a study conducted by Gagnon et al. ${ }^{18)}$, the values were $44.5 \%(\mathrm{BW})$ at the $\mathrm{TH}$ and $39.6 \%$ (BW) at the LH, similar to the values of the present study, $43.2 \%(\mathrm{BW})$ at the $\mathrm{TH}$ and $41.3 \%(\mathrm{BW})$ at the LH. Gagnon et al. advised that the maximum vertical reaction force was different between male and female spinal cord injury patients because in the case of males, force was delivered from the $\mathrm{TH}$ to the $\mathrm{LH}$ while in the case of females, force was exerted on both hands almost simultaneously because males were stronger than females ${ }^{18)}$. In the present study, since all of the subjects were males, the subjects exerted more force at the $\mathrm{TH}$ when they transferred and a relatively smaller vertical reaction force was observed at the LH. When the hand positions were low, the movement accordingly began with the trunk more bent, and this seems to have acted as a dynamic advantage for vertical reaction force in the phase of hip lifting during transfer movements.

With regard to the shearing force (anterior/posterior, medial/lateral) measured at the force plates, the anterior shearing force was found to be higher when the hand position was lower than the seat position, and the lateral shear force was found to be higher when the hand height was the same as the height of the seat. When the hand position is low, greater movement to bend the trunk forward occurs. In reaction to the forward bending of the trunk, relatively more anterior and relatively smaller lateral forces act. This is associated with the destabilizing force that decreases when the hand height decreases due to the COM becoming farther from the BOS as the hand height decreases. Duclos et al. ${ }^{11)}$ advised that if the destabilizing force is large, the movement of the center of pressure (COP) to the outside of the BOS would become more difficult, and that if the COM is high, the destabilizing force would be reduced allowing movements to be made with a smaller force. Given that when the hand is lower, the vertical reaction forces at the $\mathrm{TH}$ and the $\mathrm{LH}$ as well as the lateral force are smaller, it is our opinion that when the bearing surface for the arm is lower, transfer movements can be made with smaller force, and, in particular, the vertical loads and lateral loads on the shoulder joint can be reduced.

Limitations of the present study include the fact that the experiment was not conducted with spinal cord injury patients with shoulder pain, and that data were not obtained through measurements in actual transfer environments, but were obtained in a laboratory setting. Furthermore, since the experiment was conducted with only healthy male, the results cannot be generalized to both sexes, and even though the subjects were educated not to use their lower extremity, they might not have been able to control their lower extremities as completely as actual patients.

Taken together, our results indicate that as the hand position becomes lower, trunk flexion increases. This would act as a dynamic advantage (propulsion) for the movement to lift the hip, thereby reducing the vertical reaction force and shear force acting on the shoulder joint, thereby reducing the load on the shoulder joint.
Through the present study, the position of the supporting arms during SPT movements lower than the seat position was identified to have biomechanical advantages over the position of the supporting arms at the same height as the seat position. The results of the present study can be applied as guidelines for effective and safe methods for quadriplegia or spinal cord injury patients' SPT, and can be utilized as reference data when considering the appropriate heights of aids for wheelchairs.

\section{REFERENCES}

1) Milicevic S, Piscevic V, Bukumiric Z, et al.: Analysis of the factors influencing functional outcomes in patients with spinal cord injury. J Phys Ther Sci, 2014, 26: 67-71. [Medline] [CrossRef]

2) Allison GT, Singer KP, Marshall RN: Transfer movement strategies of individuals with spinal cord injuries. Disabil Rehabil, 1996, 18: 35-41. [Medline] [CrossRef]

3) Suyama T, Takahashi K, Shibuta H, et al.: Pain and rehabilitation in patients with spinal cord injury. J Phys Ther Sci, 2001, 13: 59-64. [CrossRef]

4) Dalyan M, Cardenas DD, Gerard B: Upper extremity pain after spinal cord injury. Spinal Cord, 1999, 37: 191-195. [Medline] [CrossRef]

5) Burnham RS, Steadward RD: Upper extremity peripheral nerve entrapments among wheelchair athletes: prevalence, location, and risk factors. Arch Phys Med Rehabil, 1994, 75: 519-524. [Medline]

6) Finley MA, McQuade KJ, Rodgers MM: Scapular kinematics during transfers in manual wheelchair users with and without shoulder impingement. Clin Biomech (Bristol, Avon), 2005, 20: 32-40. [Medline] [CrossRef]

7) Pentland WE, Twomey LT: Upper limb function in persons with long term paraplegia and implications for independence: part II. Paraplegia, 1994, 32: 219-224. [Medline] [CrossRef]

8) Gagnon D, Nadeau S, Noreau L, et al.: Trunk and upper extremity kinematics during sitting pivot transfers performed by individuals with spinal cord injury. Clin Biomech (Bristol, Avon), 2008a, 23: 279-290. [Medline] [CrossRef]

9) Gagnon D, Nadeau S, Desjardins P, et al.: Biomechanical assessment of sitting pivot transfer tasks using a newly developed instrumented transfer system among long-term wheelchair users. J Biomech, 2008b, 41: 11041110. [Medline] [CrossRef]

10) Koontz AM, Kankipati P, Lin YS, et al.: Upper limb kinetic analysis of three sitting pivot wheelchair transfer techniques. Clin Biomech (Bristol, Avon), 2011, 26: 923-929. [Medline] [CrossRef]

11) Duclos $C$, Desjardins $P$, Nadeau $S$, et al.: Destabilizing and stabilizing forces to assess equilibrium during everyday activities. J Biomech, 2009, 42: 379-382. [Medline] [CrossRef]

12) Gagnon D, Duclos C, Desjardins P, et al.: Measuring dynamic stability requirements during sitting pivot transfers using stabilizing and destabilizing forces in individuals with complete motor paraplegia. J Biomech, 2012, 45: 1554-1558. [Medline] [CrossRef]

13) Gagnon D, Koontz A, Mulroy SJ, et al.: Biomechanics of sitting pivot transfers among individuals with a spinal cord injury: a review of the current knowledge. Top Spinal Cord Inj Rehabil, 2009, 15: 33-58. [CrossRef]

14) Tanimoto $Y$, Nanba $K$, Tokuhiro A, et al.: Measurement system of transfer motion for patients with spinal cord injuries. IEEE Trans Instrum Meas, 2008, 57: 213-219. [CrossRef]

15) Forslund EB, Granström A, Levi R, et al.: Transfer from table to wheelchair in men and women with spinal cord injury: coordination of body movement and arm forces. Spinal Cord, 2007, 45: 41-48. [Medline] [CrossRef]

16) Nawoczenski DA, Clobes SM, Gore SL, et al.: Three-dimensional shoulder kinematics during a pressure relief technique and wheelchair transfer. Arch Phys Med Rehabil, 2003, 84: 1293-1300. [Medline] [CrossRef]

17) Riek LM, Ludewig PM, Nawoczenski DA: Comparative shoulder kinematics during free standing, standing depression lifts and daily functional activities in persons with paraplegia: considerations for shoulder health. Spinal Cord, 2008, 46: 335-343. [Medline] [CrossRef]

18) Gagnon D, Nadeau S, Noreau L, et al.: Comparison of peak shoulder and elbow mechanical loads during weight-relief lifts and sitting pivot transfers among manual wheelchair users with spinal cord injury. J Rehabil Res Dev, 2008c, 45: 863-873. [Medline] [CrossRef] 